\title{
A Case of Ampiginous Choroiditis
}

\author{
Pieter Lambrecht Marnix Claeys Ilse De Schryver \\ Department of Ophthalmology, Ghent University Hospital, Ghent, Belgium
}

\section{Key Words}

Ampiginous choroiditis · Relentless placoid chorioretinitis · Primary inflammatory choriocapillaropathy

\begin{abstract}
Purpose: Presentation of a case report of a unilateral ampiginous choroiditis. Methods: This is an observational case report. Results: A 70-year-old woman was referred to us with unilateral scattered chorioretinal lesions. The multifocal pattern of the lesions and the angiographic features led to the diagnosis of ampiginous choroiditis. Conclusion: Ampiginous choroiditis is a primary inflammatory choriocapillaropathy with characteristics of both acute posterior multifocal placoid pigment epitheliopathy and serpiginous choroiditis.
\end{abstract}

(C) 2015 The Author(s)

Published by S. Karger AG, Basel

\section{Case Report}

This case report is about a 70-year-old Caucasian woman who is in good general health although she has medically controlled arterial hypertension and is overweight. The patient has a 3-dpt hyperopic refraction and there are no important past general or ophthalmologic antecedents in the patient's history. The patient developed progressive painful visual blurring in the right eye during an episode of severe sinusitis. Consultation with her local ophthalmologist showed anterior chamber inflammation, macular subretinal fluid with cystoid macular oedema (fig. 1) and scattered whitish lesions in the fundus of the right eye. Ophthalmologic examination of the left eye was unremarkable. Anterior chamber inflammation disappeared after treatment with topical steroids, after which the patient was referred to us because of manifestation of new lesions and evolution of the scattered chorioretinal lesions towards atrophy. 
Lambrecht et al.: A Case of Ampiginous Choroiditis

Consultation at our hospital showed a best-corrected visual acuity of 20/40 for the right and 20/20 for the left eye. Slit lamp biomicroscopy showed bilateral nuclear sclerosis but no endothelial precipitates or posterior synechiae. Eye pressure was 12 and $14 \mathrm{~mm} \mathrm{Hg}$ in the right and left eye, respectively. Fundus examination of the right eye showed a peripapillary lesion with pseudopodial extensions and multiple yellow-greyish irregularly bordered lesions (fig. 2a). Fluorescein angiography demonstrated these lesions to be early hypofluorescent with late hyperfluorescent staining of the borders (fig. 2b). Indocyanine green (ICG) angiography demonstrated these lesions to be early and late hypofluorescent (fig. 2c). Blue light autofluorescence showed these lesions to be hypoautofluorescent with some being mildly granular hyperautofluorescent (fig. 2d). An OCT scan of the macula showed residual macular oedema. An OCT scan through these lesions displayed outer retinal atrophy. Ophthalmologic examination of the left eye was unremarkable.

Workup at her local ophthalmologist and with us showed that antinuclear factor, rheumatoid factor, angiotensin-converting enzyme, anti-proteinase 3 and anti-myeloperoxidase, calcemia and syphilis serology were all normal. There was no history of previous exposure to tuberculosis. A tuberculin skin test and chest radiography were both normal. There were no systemic signs or symptoms that were suggestive for a diagnosis of sarcoidosis. The multifocal pattern and the angiographic features led us to the diagnosis of ampiginous choroiditis. An intravitreal injection with triamcinolone acetonide was given as a treatment for the macular oedema with resolution of the macular oedema as a consequence (fig. 3). We started immunomodulatory treatment with azathioprine $2 \times 50 \mathrm{mg} /$ day to prevent recurrence of inflammation. After 4 months, we decided to stop therapy with azathioprine since it was not well tolerated by the patient. At the time of writing this case report, the patient has been followed for more than 1 year. During this period, visual acuity has remained stable and no appearance of new lesions or enlargement of the known lesions has occurred.

\section{Discussion}

We present a case report of unilateral uveitis in a healthy 70-year-old woman. Visual symptoms and eye pain developed during an episode of sinusitis. Consultation with her local ophthalmologist showed scattered retinal lesions reminiscent of acute posterior multifocal placoid pigment epitheliopathy (APMPPE). These lesions, however, evolved to outer retinal atrophy with angiographic characteristics reminiscent of serpiginous choroiditis. The combination of characteristics of both APMPPE and serpiginous choroiditis led to the diagnosis of ampiginous choroiditis [1]. A similar disease entity has been called relentless placoid chorioretinitis [2]. Diagnostic criteria used by Jyotirmay et al. [3] help to delineate this disease. The clinical profile of 16 patients with ampiginous choroiditis showed a male preponderance (7:3), a median age of 34 years, $81 \%$ bilateral involvement and $35 \%$ suffered from recurrences [3].

APMPPE and serpiginous choroiditis are both classified as a primary inflammatory choriocapillaropathy [4]. Inflammation at the level of the choriocapillaris leads to areas of choriocapillaris non- or hypoperfusion with ischaemic consequences both at the level of the choroid and at the level of the outer retina. Rarely, an intermediary form between these two diseases, i.e. ampiginous choroiditis, can be seen, but the reason why some patients develop a blend of both diseases is unknown. It has been proposed that the behaviour of each type of primary inflammatory choriocapillaropathy has to do with the level and severity of vascular damage to the choriocapillaris [4]. An argument used in favour of this proposition is the fact that in a large series of serpiginous choroiditis, some lesions had initial features of APMPPE 
Lambrecht et al.: A Case of Ampiginous Choroiditis

but showed progression towards serpiginous choroiditis during follow-up [5]. The aetiology of serpiginous choroiditis is unclear, with degenerative, autoimmune and/or infectious processes considered [6]. The aetiology of ampiginous choroiditis, which is considered by some to be a subclass of serpiginous choroiditis, is likewise unclear, with some speculating it to be an ocular manifestation of a larger systemic autoimmune condition [3]. A case of ampiginous choroiditis following a human papillomavirus vaccine was reported, although a causal relationship is not sure [7]. An ampiginous-like case report showed positive serology in blood and polymerase chain reaction (PCR) in aqueous humour for Toxoplasma gondii infection. That patient also had a tuberculin skin test with $15 \mathrm{~mm}$ of induration, but negative aqueous humour PCR for Mycobacterium tuberculosis [8]. Our patients' workup could not identify an underlying cause for this disease, making the origin of this disease unclear. Since our patient developed symptoms during an episode of sinusitis, it is tempting to suspect an infectious trigger as a cause.

An important differential diagnostic of ampiginous choroiditis is multifocal serpiginoid choroiditis caused by Mycobacterium tuberculosis, Treponema pallidum or a herpesvirus [6]. Syphilis was excluded as a cause since syphilis serology was negative. Mycobacterium tuberculosis infection is unlikely since there was no history of previous exposure to tuberculosis and since a tuberculin skin test and chest radiography were both negative [9]. Multifocal lesions, unilaterality and considerable vitreous or anterior chamber inflammation, as in our patient, point towards a diagnosis of tuberculosis multifocal serpiginoid choroiditis instead of a serpiginous choroiditis [6]. Peripapillary involvement from the early phases of the disease, as in our patient, is, however, not typical for a tuberculosis multifocal serpiginoid choroiditis [6]. We did not perform an anterior chamber tap to do a PCR for Mycobacterium tuberculosis or herpesviruses since the patient did not consult us during active inflammation, making a positive PCR result more unlikely. Two other important differential diagnoses are sarcoidosis and granulomatosis with polyangiitis, formerly known as Wegener's granulomatosis. The patient had no systemic manifestations that were suggestive for sarcoidosis. Workup with serum angiotensin-converting enzyme, serum calcium and a chest radiograph also showed no arguments for sarcoidosis. Granulomatosis with polyangiitis is unlikely since the patient did not have systemic manifestations suggestive of this disease and since antiproteinase 3 and anti-myeloperoxidase testing were normal.

We chose to treat the macular oedema with an intravitreal injection with triamcinolone acetonide. We started immunomodulatory therapy with azathioprine to prevent recurrences, but after 4 months, this medication was stopped since the patient did not tolerate it well. After consulting the patient, we decided not to start another immunomodulatory agent because there are indications that only one third of patients suffer relapses [3]. After having followed up this patient for more than 1 year, we have not seen an evolution in number or size of retinal lesions. The absence of diffuse choroidal hyperfluorescence with ICG angiography at the edges of the lesions indicates no progression of the lesions [4]. Since some of these lesions still have a hyperautofluorescent aspect, we do suspect some smouldering residual activity in these lesions. We plan to follow up our patient closely and in the event of any development of activity of an ampiginous lesion towards the macula, we will start systemic therapy with corticosteroids and immunomodulatory therapy. 
Lambrecht et al:: A Case of Ampiginous Choroiditis

\section{Statement of Ethics}

The patient has given voluntary written informed consent. During treatment, the health of the patient was our first consideration and we have acted in the patient's best interest when providing medical care.

\section{Disclosure Statement}

The authors declare no conflicts of interest. There are no financial or nonfinancial interests/relationships that may be interpreted to have influenced the manuscript.

\section{References}

1 Nussenblatt RB: Serpiginous choroidopathy; in Nussenblatt RB, Whitcup SM (eds): Uveitis, ed 4. Edinburgh, Mosby, 2010, pp 373-382.

-2 Jones BE, Jampol LM, Yannuzzi LA, Tittl M, Johnson MW, Han DP, Davis JL, Williams DF: Relentless placoid chorioretinitis: a new entity or an unusual variant of serpiginous chorioretinitis? Arch Ophthalmol 2000;118:931-938.

-3 Jyotirmay B, Jafferji SS, Sudharshan S, Kalpana B: Clinical profile, treatment, and visual outcome of ampiginous choroiditis. Ocul Immunol Inflamm 2010;18:46-51.

4 Cimino L, Mantovani A, Herbort C: Primary inflammatory choriocapillaropathies; in Krieglstein G, Weinreb R, Pleyer U, Mondino B (eds): Uveitis and Immunological Disorders. Berlin, Springer, 2005, pp 209-231.

5 Gupta V, Agarwal A, Gupta A, Bambery P, Narang S: Clinical characteristics of serpiginous choroidopathy in North India. Am J Ophthalmol 2002;134:47-56.

6 Nazari Khanamiri H, Rao NA: Serpiginous choroiditis and infectious multifocal serpiginoid choroiditis. Surv Ophthalmol 2013;58:203-232.

-7 Khalifa YM, Monahan PM, Acharya NR: Ampiginous choroiditis following quadrivalent human papilloma virus vaccine. Br J Ophthalmol 2010;94:137-139.

-8 Mahendradas P, Kamath G, Mahalakshmi B, Shetty KB: Serpiginous choroiditis-like picture due to ocular toxoplasmosis. Ocul Immunol Inflamm 2007;15:127-130.

-9 Vasconcelos-Santos DV, Zierhut M, Rao NA: Strengths and weaknesses of diagnostic tools for tuberculous uveitis. Ocul Immunol Inflamm 2009;17:351-355.

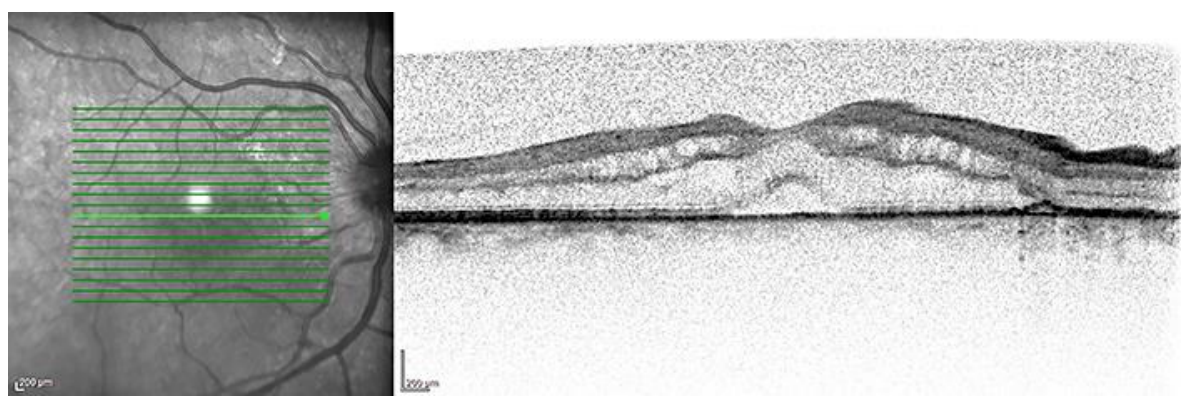

Fig. 1. OCT scan of the macula of the right eye at the time of presentation. 
Lambrecht et al:: A Case of Ampiginous Choroiditis
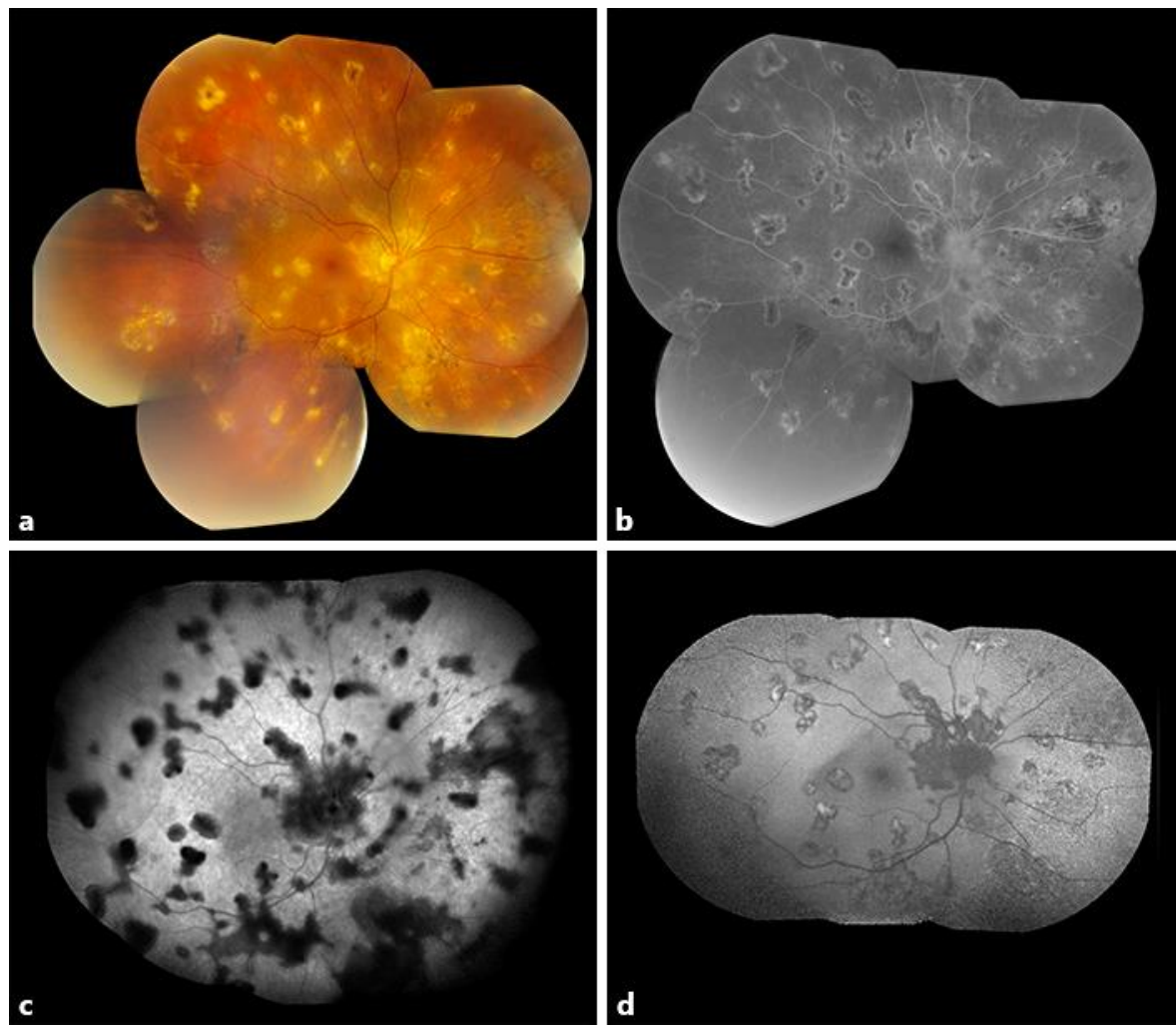

Fig. 2. Fundus pictures of the right eye at the time of referral. a Colour picture. b Fluorescein angiography. c ICG angiography. d Blue light autofluorescence.

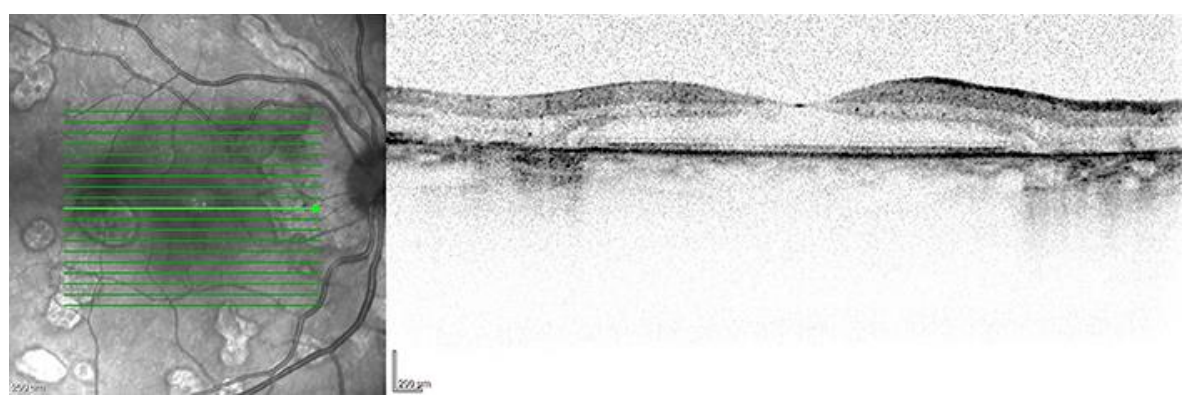

Fig. 3. OCT scan of the macula of the right eye after treatment with intravitreal triamcinolone acetonide. 